\title{
Diagnóstico y tratamiento de la plagiocefalia posicional. Protocolo para un Sistema Público de Salud
}

\author{
J. Esparza; J. Hinojosa; Mª. Muñoz; A. Romance*; I. García-Recuero* y A. Muñoz**
}

Unidad de Cirugía Craneofacial (Servicios de Neurocirugía Pediátrica, Cirugía Maxilofacial* y Neurorradiología**). Hospital Infantil 12 de Octubre. Madrid.

Resumen

Introducción. La plagiocefalia posicional es actualmente la causa más frecuente de asistencia en una consulta de neurocirugía pediátrica, tanto en España como en todos los países occidentales. A ello se suma la considerable confusión existente en la literatura en relación a aspectos como la terminología, conceptos fisiopatológicos, diagnóstico diferencial con la craneosinostosis y por supuesto en el tratamiento más adecuado a seguir.

Objetivos. Intentar clarificar estos conceptos y además presentar un protocolo de asistencia que nos ha solicitado recientemente la Administración Sanitaria de la Comunidad de Madrid.

Protocolo. Pretende lograr la coordinación entre pediatras y neurocirujanos, así como conseguir una información precisa de los principales datos de esta patología para los familiares, pediatras y neurocirujanos.

Material y métodos. Se establecen una serie de datos de consenso. Los niños son clasificados en tres grados de deformación (leve, moderada y grave) según los índices medidos en fotografías digitales.

Además el proceso del diagnóstico y tratamiento tiene dos fases: fase pediátrica (hasta los $\mathbf{5}$ meses de edad) y fase neuroquirúrgica (desde los 5 meses de edad). Los niños serán enviados a Neurocirugía después de haber sido tratados con tratamiento posicional y rehabilitación y solamente a partir de los 5 meses de edad.

Las razones de todo ello son explicadas en el protocolo que define también las funciones y responsabilidades de cada especialista.

El tratamiento que se propone es escalonado, comenzando por las medidas posicionales y de rehabilitación, seguidas de ortesis craneal y en último lugar del tratamiento quirúrgico.
PALABRAS CLAVE: Plagiocefalia. Craneosinostosis occipital. Torticollis. Ortesis raneal.

Positional plagiocephaly. Diagnosis and treatment

Summary

Introduction. Positional plagiocephaly is nowadays the most frequent consultation at pediatric neurosurgical departments in Spain and western countries. Another important issue is the confusion existing in literature regarding terminology, physiopathology, differential diagnosis with true synostosis and, of course, proper recommendations for treatment.

Objectives. To clarify all these concepts and present a protocol that was recently asked by the Health Administration of the Community of Madrid.

Protocol. Pretends to achieve coordination among paediatricians and neurosurgeons, as much as to offer precise information about data concerning this entity for parents, paediatricians and neurosurgeons.

Material and methods. Previous consensus is reached about some data. Children are classified in three categories of deformation (mild; moderate; severe), according to measurements on digital photography.

Diagnosis and treatment follows two phases: Paediatric phase (up to 5 months of age) and Neurosurgical phase (from 5 months on). Children would be referred to neurosurgical consultation only after being treated with postural changes and physiotherapy by the age of 5 months. Reasons are explained in the protocol that defines also functions and responsibilities for each speciality.

Conclusion. Treatment proposed here is staged, starting with postural changes and physiotherapy, followed by orthotic cranial devices and finally surgical treatment.

KEY WORDS. Plagiocephaly. Lambdoid craniosynostosis. Torticollis. Headband. 


\section{Introducción}

Antecedentes. Durante el año 1992 se desarrolló por parte de la Asociación Americana de Pediatría (AAP) una campaña de divulgación conocida como "Back to sleep" para intentar disminuir el número de casos de "muerte súbita del lactante”. En esta campaña se recomendaba que los niños fueran colocados boca arriba para dormir. Como resultado, algunas estadísticas destacan que se consiguió disminuir la mortalidad hasta en un $40 \%$, por lo que actualmente no parece aconsejable cambiar una estrategia que ha tenido tanto éxito ${ }^{1,2}$.

Sin embargo y ya por la misma época, se comenzaron a publicar en USA los primeros datos del alarmante aumento de las deformaciones craneales conocidas como "plagiocefalias posicionales posteriores u occipitales" y que sin duda está en relación con esta campaña y, por consiguiente, con el hábito ya bien establecido de que los lactantes duerman sobre sus espaldas. La incidencia de estas deformaciones craneales seguramente ha aumentado en la actualidad, al estar la población más alertada sobre el problema de la muerte súbita, pero sin la contrapartida de tener suficiente información para prevenir el desarrollo de la plagiocefalia ${ }^{3,13}$.

Un factor muy importante que ha aumentado el desconcierto general es la confusión de terminología y conceptos básicos que sobre este problema existe en la literatura ${ }^{25}$, lo que seguramente ha impedido que se desarrollen estrategias adecuadas.

Actualmente, la plagiocefalia posicional es sin duda la causa más frecuente de asistencia en una consulta de neurocirugía pediátrica en nuestro medio. En el Hospital Infantil 12 de Octubre y en los últimos años la afluencia de niños ha sido tan masiva que hemos tenido que habilitar una consulta especial para esta patología. En esta consulta, que funciona desde el año 1998, se atienden actualmente unos 1000 niños anuales. Fruto de esta experiencia fue el primer trabajo publicado ya en al año 2000 por nosotros, que también fue el primero en España y además en esta misma revista ${ }^{23}$, sobre tratamiento con ortesis craneal.

Es además previsible un incremento de esta demanda en el futuro, debido a una evidente falta de estrategia en la asistencia pediátrica, que coincide con una presión social creciente por parte de la población.

$\mathrm{Si}$ bien no hay evidencia científica actualmente que demuestre que la plagiocefalia posicional produzca otras alteraciones que no sean las de la deformación craneal y/o facial ${ }^{24,26}$, estas deformidades pueden ser tan aparatosas que es comprensible la grave preocupación que ocasiona en muchas familias.

Objetivos. Intentar clarificar todo lo posible la confusión existente sobre esta patología y presentar un protocolo de asistencia que nos ha sido solicitado recientemente por la Administración Sanitaria de la Comunidad de Madrid.

En este protocolo se intenta coordinar las funciones y responsabilidades de los actores principales en todo el proceso diagnóstico y terapéutico: pediatras, rehabilitadores, neurocirujanos pediátricos y las familias de los niños afectados.

\section{Datos previos para establecer un "consenso"}

Durante demasiados años, los conceptos fundamentales para entender estas deformidades han sido extraordinariamente confusos ${ }^{25}$ aunque ha habido recientemente algunos autores que han intentado clarificar sus aspectos más importantes ${ }^{7,9,8,12,16,24,25,27}$.

Sin duda la mayor dificultad ha sido el diagnóstico diferencial de la plagiocefalia posicional "no craneosinostótica" con los casos de "auténtica craneosinostosis occipital”.

Para este trabajo creemos haber revisado la principal literatura publicada sobre el tema y también nos hemos basado en nuestra propia experiencia de miles de casos de plagiocefalia posicional tratados en nuestra Unidad.

Definición y datos generales. La plagiocefalia posicional es conocida también en la literatura por otros términos como por ejemplo: plagiocefalia por moldeamiento, plagiocefalia occipital, plagiocefalia deformativa, plagiocefalia sin craneosinostosis, plagiocefalia postural, plagiocefalia funcional y plagiocefalia posterior.

En este trabajo la denominaremos plagiocefalia posicional, porque probablemente es el que menos confusión genera.

Atendiendo a la etiología de la deformación, la plagiocefalia posicional es de "carácter externo" porque está producida por fuerzas mecánicas externas que actúan sobre la sutura lambdoidea o la región posterior del cráneo, bien sea durante la vida intrauterina o posteriormente $e^{7,9,12,20,22,24,25}$, a diferencia de la plagiocefalia craneosinostótica, que como todas las craneosinostosis es debida a factores intrínsecos que afectan a las propias suturas craneales (suturas lambdoideas).

Diversos factores pueden actuar sobre la cabeza fetal produciendo un fenómeno de moldeamiento craneal: posiciones fetales prolongadas, embarazos múltiples, anomalías uterinas (útero bicorne), macrocefalia, grandes fetos, partos con fórceps o ventosas.. etc.

Después del nacimiento son también muy numerosas las causas que pueden ocasionar esta deformación por moldeamiento ${ }^{7,22,24}$ : una posición elegida por el lactante sin una razón clara, el apoyo sistemático de la cabeza en la región occipital de un lado o bilateralmente, durante el sueño o en períodos de despertar, la utilización constante de los modernos "carritos" portadores de los lactantes con el niño apoyando siempre la cabeza de la misma 
forma $^{7}$, la presencia de tortícolis debido a muy diversas etiologías, lesiones en los nervios oculomotores como el $\mathrm{IV}^{\mathrm{o}}$ par o de la musculatura ocular y por último numerosas lesiones, incluso cerebrales, que favorecen la aparición de una plagiocefalia al disminuir la motilidad espontánea del niño.

Un mecanismo frecuente en la clínica es de carácter mixto: niños que ya nacen con asimetría craneal producida por moldeamiento intrauterino o durante el parto $\mathrm{y}$ que durante las primeras semanas de vida empeoran por asociarse cualquiera de los factores previamente citados, especialmente porque la cabeza siempre tiende a apoyarse permanentemente sobre el lado previamente aplanado, agravándose la situación todavía más cuando existe tortícolis.

En la actualidad la incidencia real de la plagiocefalia posicional es imposible de establecer, pero hay trabajos recientes que indican que si los criterios diagnósticos empleados no son correctos, la cifra puede llegar hasta un $48 \%$ de niños sanos por debajo del año de edad ${ }^{3,13}$. Por el contrario la incidencia de la craneosinostosis occipital en la literatura es muchísimo menor, quizá con una cifra de 3/100.000 nacimientos $(0.003 \%)^{26}$.

En nuestra Unidad de Cirugía Craneofacial y en el período de tiempo transcurrido entre los años 1989 a 2005, hemos operado 507 niños de muy diversas formas de craneosinostosis, habiendo entre ellos solamente 11 casos de plagiocefalia occipital (2.16\%), lo que coincide plenamente con las cifras aportadas por la gran mayoría de los autores $^{8,12,22,26}$.

Por otra parte la plagiocefalia posicional puede ser unilateral o también bilateral (paquicefalia) en un 20\% de los casos $^{11}$.

La historia natural de esta deformidad es difícil de establecer claramente ${ }^{24,26}$, pero puede suponerse que en la adolescencia o la edad adulta un número indeterminado de estos niños pueden presentar alguna deformidad craneal o facial, generalmente en grados menores ${ }^{24}$.

\section{Diagnóstico clínico}

El principal diagnóstico diferencial debe realizarse con la craneosinostosis occipital.

Plagiocefalia posicional. Los datos clínicos más característicos son debidos al aplanamiento de la región parieto-occipital que hace que el peñasco se desplace anteriormente y hacia abajo y al mismo tiempo la región frontal homolateral se adelanta por crecimiento compensatorio. Así pues se producirá:

- Aplanamiento y también alopecia de la región occipital e incluso parietal.

- Abombamiento de la región occipital contralateral . Es posible también abombamiento parietal.

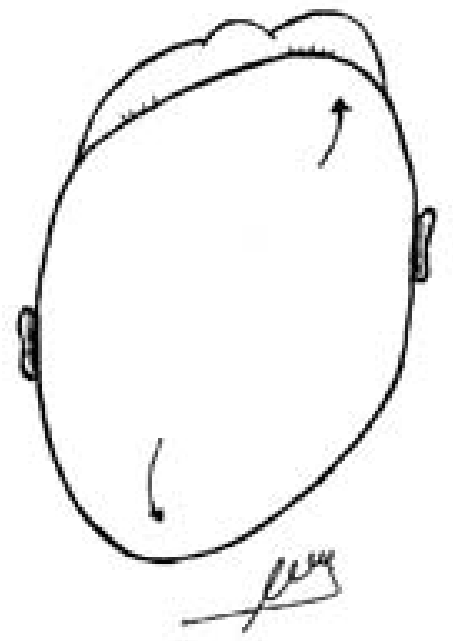

Figura 1. Esquema de Plagiocefalia posicional derecha. La cabeza forma un paralelogramo: la región occipital deformada está aplanada o incluso “hundida”. La región occipital contralateral está abombada. La región parietal unilateral puede también estar abombada así como también la región frontal unilateral. El pabellón auricular puede estar adelantado en el lado de la lesión e incluso el zigoma también puede protuir moderadamente en el mismo lado. La raíz nasal suele estar centrada.

- Pabellón auricular ipsilateral adelantado y descendido.

- Frontal homolateral abombado.

- Raíz nasal centrada.

- Región maxilar homolateral puede estar también abombada.

La forma del cráneo es la de un paralelogramo (Fig.1). La plagiocefalia posicional se puede acompañar además de otra serie de lesiones: tortícolis frecuente, hasta en un $41 \%^{7}$ para algunos autores, macrocefalia moderada (40\%) con acúmulos extraaxiales de LCR en un $35 \% \%^{7,17}$ y muy variadas lesiones cerebrales como hidrocefalia, hemorragia perinatal, infecciones, espina bífida.. etc. hasta en un $20 \%$ de los casos ${ }^{7}$.

En algunas series también se describe algún grado de retraso psicomotor hasta en un $19 \%{ }^{7}$ o de dificultades en el aprendizaje $^{19}$.

Cuando la plagiocefalia posicional es bilateral, la cabeza adopta un aspecto braquicefálico y es conocida también con el nombre de paquicefalia ${ }^{8}$ (Fig.2). El aplanamiento afecta a ambas regiones occipitales, con aumento del diámetro vertical del cráneo en la región parietal, acentuándose la protusión del vértex y existiendo además una disminución del diámetro antero-posterior craneal. La morfología facial no se afecta, pero vista de perfil la cabeza en los casos más graves puede parecer que la región occipital ha sido "cortada por un hacha”. 
Por todo ello, la técnica de elección es la TAC craneal y la reconstrucción tridimensional ${ }^{16}$. En los casos de craneosinostosis el aspecto del cráneo suele ser trapezoidal, existiendo además una repercusión más evidente del proceso en la región mastoidea (abombamiento), así como una clara afectación de la base craneal con distorsión del eje anteroposterior $^{27}$. (Fig.10 y 11).

\section{Tratamiento}

El tratamiento de la plagiocefalia posicional consiste en una serie de medidas que en opinión de muchos autores deben ser escalonadas: rehabilitación, técnicas de ortesis craneal y la reconstrucción quirúrgica en último lugar ${ }^{20,21,22,25}$.

Durante todo el proceso, la información a las familias proporcionada por el pediatra y/o el rehabilitador debe ser lo más clara posible, educándoles especialmente en las medidas encaminadas a lograr una correcta rehabilitación posicional del niño.

Así, por ejemplo, los cambios de posición lateral de la cabeza mientras el niño duerme, con ayuda de la inclinación del colchón, el aprovechamiento del tiempo en que el niño esté despierto para que practique movimientos de la cabeza y ejercicios en superficies duras y por supuesto el tratamiento del tortícolis con adecuados movimientos del cuello son todas ellas medidas fundamentales con las que los niños se curan en la gran mayoría de los casos durante los primeros meses de vida. Son los propios padres quienes deben realizar estos ejercicios al niño, para lo que deben ser instruídos adecuadamente por el pediatra o rehabilitador (Fig. 3 y 4).

El tratamiento con ortesis craneal, aunque haya sido cuestionado por algunos autores ${ }^{5,21,25}$ parece adecuado utilizarlo en determinados casos, especialmente en los casos refractarios a anteriores tratamientos, según una opinión generalizada que resulta mayoritaria ${ }^{24}$. Estos procedimientos utilizan en realidad los mismos métodos empleados en algunas civilizaciones antiguas para lograr una modelación craneal en los lactantes, con objeto de obtener una morfología craneal previamente determinada. De la misma manera, en la actualidad, están descritos diferentes tipos de "bandas o cascos" que pretenden modificar en el plazo de unos meses la morfología del cráneo ${ }^{14,22,23}$.

Parece que la respuesta más idónea a este tipo de tratamiento puede ser obtenida a partir de los 4 meses de edad y hasta un límite de 12 meses, más allá de los cuales el cráneo deja de ser susceptible a moldeamientos externos. En todo caso y dado que este tratamiento genera unos costes evidentes, bien para las familias o en el caso de que exista financiación pública para la Administración, conviene sistematizar lo mejor posible su empleo.

Finalmente el tratamiento quirúrgico hay que reservarlo para los casos de craneosinostosis o bien para aquellos raros pacientes en los que los tratamientos anteriores no 


\section{Hoja informativa para padres y familiares}

Durante las primeras seis semanas de vida, los niños pueden tener una forma anormal de la cabeza debido a su paso por el canal del parto. Pasado este tiempo la cabeza suele recuperar un aspecto normal.

En los niños las deformidades de la cabeza más frecuentes son las llamadas posturales. En estas deformidades, las suturas ( los huesos que forman el cráneo están unidos por un tejido que forma las suturas y que les permite estar flotando para dejar al cerebro crecer durante los primeros meses de vida) están abiertas permitiendo que el cerebro crezca sin que la deformación le produzca ningún daño.

Las causas de estos cambios en la forma de la cabeza del niño son debidas a presiones ejercidas sobre los hueso del cráneo durante el embarazo y en otras ocasiones a partos difíciles, tortícolis o en la mayoría de las veces a dormir siempre sobre el mismo lado. Es en estas ocasiones cuando frecuentemente la parte posterior de la cabeza del niño (región occipital) comienza a aplanarse bien en un lado o en los dos. Esta deformación puede también acompañarse de un adelantamiento del pabellón auricular del mismo lado, así como también de la frente e incluso de la mejilla.

\section{¿Qué hacer para evitarlas?}

\section{Cambios posturales en la cuna.}

¿Ypara mejorarlas? Se deben de realizar una serie de sencillas medidas que en la gran mayoría de los casos van a curar al niño:

- Poner juguetes en el lado contrario al "plano" para que gire la cabeza.

- Colocar la cuna de tal forma que al niño se le estimule y hable desde el lado contrario al aplanado.

- Levantar el colchón poniendo una toalla o sábana hecha un rollo entre el propio colchón y el somier para inclinarlo.

- Permitir que el niño juegue sobre su "barriguita" durante los 3-4 meses primeros, siempre en presencia de los padres para fortalecer los músculos cervicales.

- En casos de dudas, consulte siempre con su pediatra.

Figura 3. Hoja Informativa para las familias.

hayan tenido el efecto deseado $0^{7,21,24,25}$.

Protocolo asistencial. -Los neonatos que nacen ya con plagiocefalia posicional son excluídos automáticamente de este protocolo. En efecto, todo niño que ha desarrollado una plagiocefalia intrauterina debe ser referido a un especialista para un diagnóstico diferencial (excluir craneosinostosis u otras malformaciones).

El protocolo se basa en la siguiente premisas:

- Edad del niño. La mayoría de estos niños pueden curarse con medidas posicionales practicadas por su familia y dirigida por el pediatra. Así pues el tratamiento posicional es fundamentalmente eficaz antes de los 5 meses de edad aproximadamente. Posteriormente, el niño es capaz ya de mover la cabeza por sí mismo e incluso sostenerla y por lo tanto las medidas posicionales son bastante más difíciles.

Por otra parte hay bastante consenso en establecer que la edad adecuada para el tratamiento con ortesis craneal, en lo casos en que esté indicado, es a partir de los 4-5 meses de edad. Por lo tanto, la edad que hemos elegido para dividir este protocolo en dos fases es la de 5 meses.

- El diagnóstico diferencial definitivo y el tratamiento con ortesis craneal o con cirugía, en los casos en que sea necesario, debería ser realizado por expertos en deforma- 


\section{Cuidados del recién nacido para evitar las deformidades craneales}
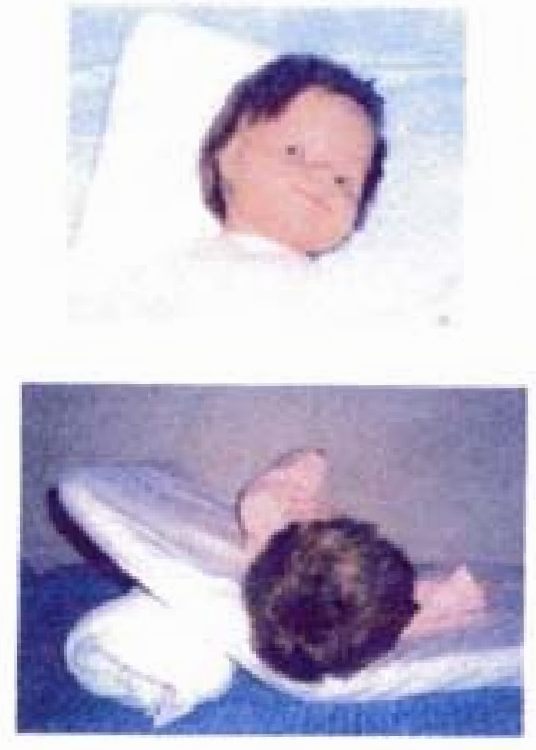

Pero si se produce una deformación postural el niño deberá dormir inclinado sobre el lado contrario. Para ello se pondrá un rodete entre el colchón y el somier de la cuna.

Para que la forma del cráneo sea
normal debemos realizar cambios en la posición de la cabeza durante los cuatro primeros meses de vida.

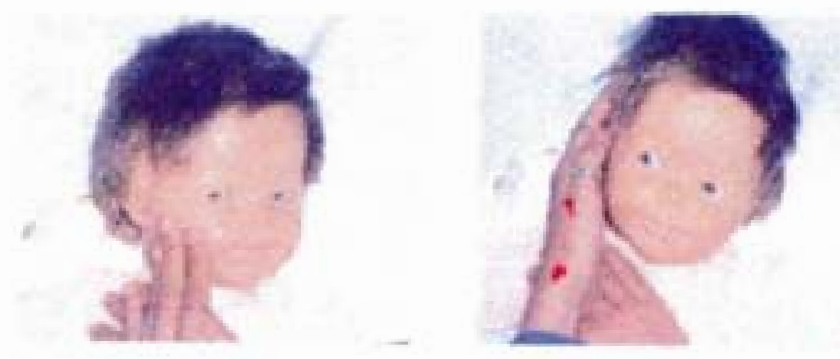

Si existe una contractura cervical será necesaria la realización diaria de los siguientes ejercicios: $1 \%$ rotación de la cabeza durante unos segundos a cada lado. $2 \%$ movimientos suaves de inclinación lateral.

También se pondrá al niño "boca abajo" para fortalecer la musculatura cervical. Siempre en presencia de los padres y durante unos minutos.

Figura 4. Hoja Informativa para tratamiento posicional 
ciones craneales como son los especialistas en Neurocirugía Pediátrica.

Por todo lo anterior el proceso tiene dos fases:

- Fase pediátrica (hasta los 5 meses de edad).

- Fase neuroquirúrgica. (desde los 5 meses hasta el año o año y medio de edad).

NOTA MUY IMPORTANTE: En la transición entre una fase y otra, es decir, cuando el pediatra decide la referencia del niño al neurocirujano pediátrico, no son admisibles los RETRASOS BUROCRATICOS, dado que la edad de tratamiento eficaz puede sobrepasarse si estos retrasos existen.

\section{Fase Pediátrica (hasta los 5 meses)}

Aunque el niño duerma sobre su espalda, el pediatra aconsejará siempre los cambios periódicos de posición de la cabeza de manera preventiva. Estos cuidados se intensificarán en los casos en los que aparezcan los primeros signos de plagiocefalia (Fig.1). La información familiar puede complementarse con los documentos que se adjuntan (Fig. 3 y 4$)$.

Durante esta fase no son necesarios los estudios radiológicos, aunque sería aconsejable que el pediatra realizara mediciones con fotografía digital para clasificar la gravedad del caso y calcular el Î́ndice de Asimetría Craneal, según se explica en las figuras 5, 6 y 7 . Dado que en los niños puede ser a veces difícil obtener una buena fotografía, pueden emplearse cualquiera de las mediciones explicadas en las figuras para los casos de plagiocefalia unilateral.

En los casos con plagiocefalia posicional bilateral debe emplearse el índice A-P-Lateral de la fig. 8.

En todos los casos se establecerá una valoración del grado de deformidad, clasificando los casos en tres grados: grave, moderado y leve, según las distancias obtenidas en las fotografías.

Esto no implica que los niños con grados más graves se envíen inmediatamente al neurocirujano, puesto que incluso estos niños tienen muchas posibilidades de mejorar o curarse con el tratamiento posicional.

Por otra parte, el pediatra debe investigar la presencia de tortícolis para comenzar enseguida el tratamiento. Si es muy grave, el niño debe enviarse a una Unidad de Rehabilitación.

Si a los 5 meses de edad y a pesar de haber seguido todo el tratamiento anterior de una forma rigurosa, el niño no ha mejorado lo suficiente como para pensar en una buena evolución, se enviará a Neurocirugía Pediátrica.

Fase Neuroquirúrgica desde los 5 meses hasta el año y medio de edad).

El neurocirujano valorará en primer lugar si el niño

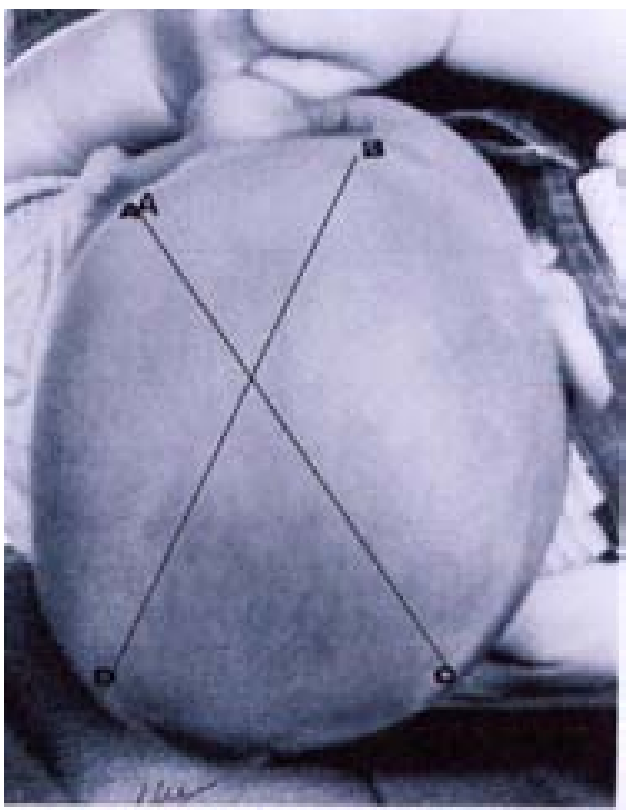

Figura 5. Índice de asimetría craneal. Fotografía digital. Diagonal mayor (BD): línea trazada desde el borde externo de la órbita hasta la región occipital abombada, obteniéndose la distancia máxima en mm. Diagonal menor (AC), distancia mínima desde el borde externo de la órbita hasta el occipital aplanado. Indice = diagonal mayor - Diagonal menor (mm). Grado de deformidad. Leve: 010mm. Moderada: 10-20 mm. Grave: superior a $20 \mathrm{~mm}$

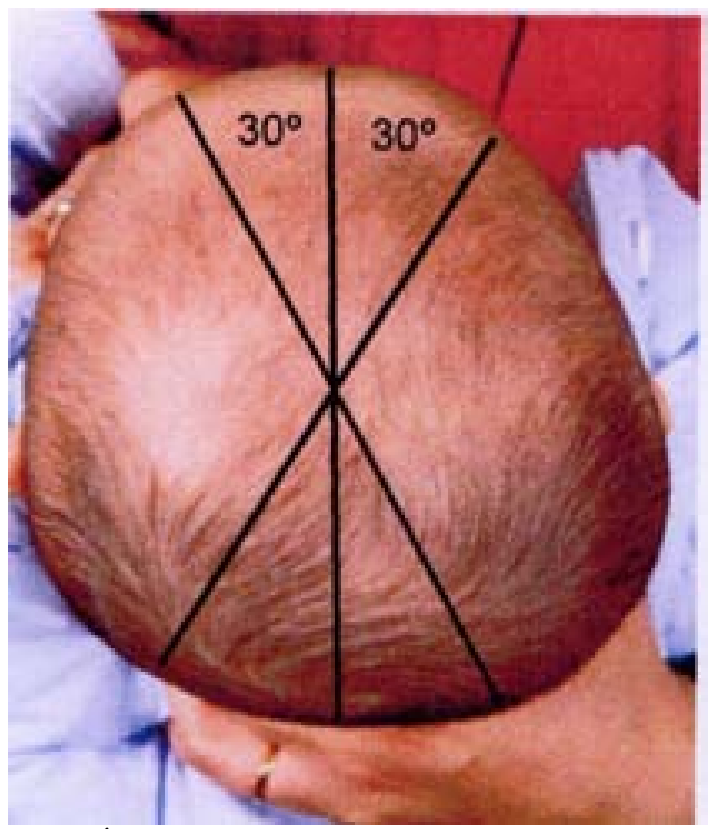

Figura 6. Índice de asimetría craneal: distancia obtenida trazando una línea a $30^{\circ}$ de la línea media. Distancia obtenida de la misma manera en el lado aplanado. Grado de deformidad. Leve: 0-10mm. Moderada: 10-20 mm. Grave: superior a $20 \mathrm{~mm}$. 


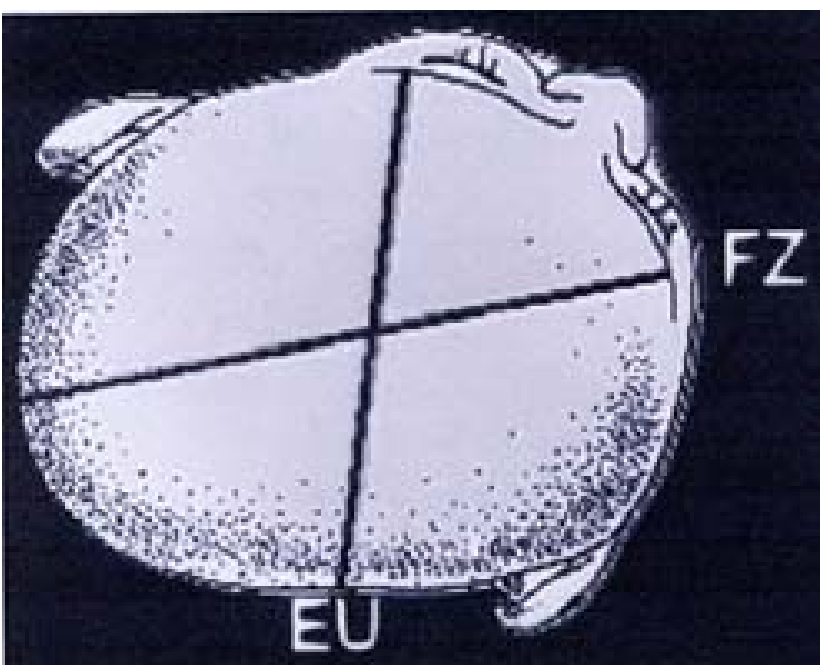

Figura 7. Indice de Asimetría Craneal. FZ: distancia máxima obtenida desde la unión frontozigomática (FZ) hasta la región occipital abombada. EU: distancia mínima obtenida desde la unión frontozigomática hasta el Eurion (unión occipito-parietal). Indice de Asimetría: FZmm-EU $\mathrm{mm}$. Grado de deformidad: Leve: 0-10mm. Moderada: 1020. Grave: superior a $20 \mathrm{~mm}$.

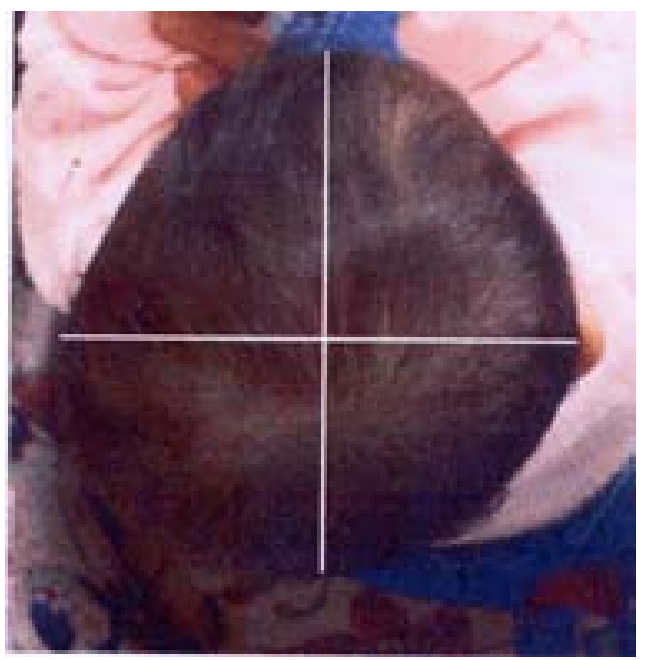

Figura 8. Indice Cefalométrico (A-P-Lateral): Cociente entre la distancia biparietal máxima dividida por la distancia Ant-Post tomada en la línea media y multiplicado por 100 en mm. Indice=Biparietal/A-P x $100 \mathrm{~mm}$. Grado de deformidad: Leve: 80-90 mm. Moderada: 90-100 mm. Grave: Mayor de $100 \mathrm{~mm}$.

ha seguido un tratamiento posicional correcto. Podrá ser referido de nuevo al pediatra si el niño no ha cumplido los requisitos de la primera fase y no se cree indicado un casco ortopédico.

Valorará todo el caso estableciendo un diagnóstico, posibilidades que le quedan al tratamiento posicional y necesidad de estudios radiológicos.
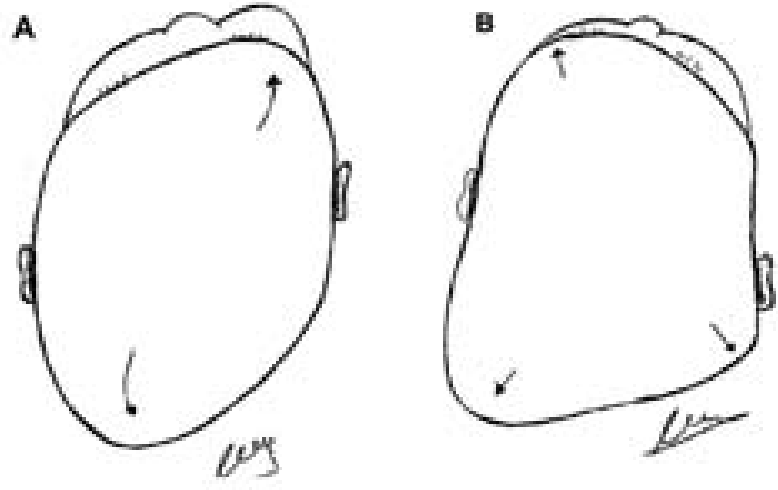

Figuras 9 y 10. Diagnóstico diferencial con la craneosinostosis occipital.

Figura 9. A) Plagiocefalia posicional: aspecto del cráneo en paralelogramo. B) Craneosinostosis: aspecto trapezoidal del cráneo por abombamiento de la región mastoidea.
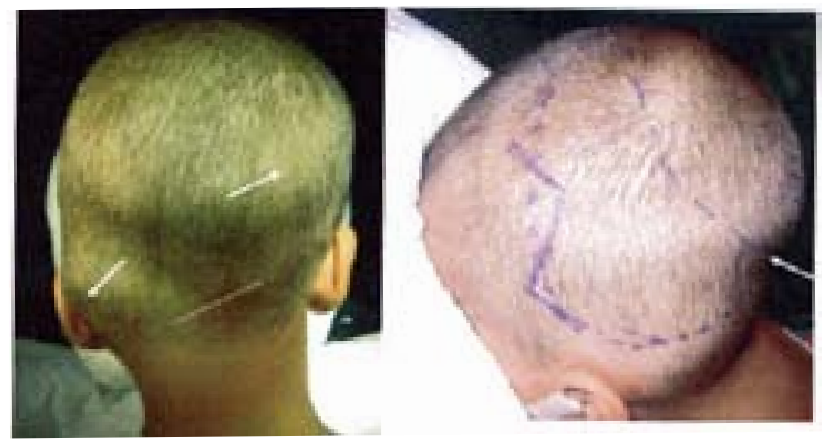

Figura 10. Diagnóstico diferencial con la craneosinostosis occipital . Plagiocefalia por craneosinostosis: crecimiento compensador parietal alto contralateral a la sutura patológica y en dirección occipito-mastoidea ipsilateral (flechas). La línea que une ambas mastoides es oblicua en dirección a la sutura. Clara estenosis y aplanamiento muy circunscrito a la región lambdoidea. Aspecto trapezoidal.

Para la clasificación de la gravedad del caso bastará con una fotografía digital, utilizándose de nuevo los índices explicados en las fig. 5, 6 y 7 para las plagiocefalias unilaterales y el índice cefalométrico de la Fig. 8 para las bilaterales.

Se valorará también de manera especial la existencia de deformación en la región craneofacial.

No es necesario realizar radiografías o TAC para las mediciones. Es suficiente una fotografía digital.

En los casos graves que no hayan mejorado con el tratamiento posicional se realizará preferentemente una TAC craneal y reconstrucción tridimensional para asegurar el diagnóstico diferencial con las craneosinostosis (Fig.9 y 10).

- Por otra parte, en aquellos niños que presenten todavía un tortícolis se seguirá con el tratamiento rehabilitador y en 
los casos de mayor gravedad que no hayan mejorado con el tratamiento se realizarán estudio radiológicos del raquis cervical y charnela cervico-occipital para descartar malformaciones. En los casos más reacios se valorará también la posibilidad de indicación quirúrgica del tortícolis (intervenciones sobre los músculos contracturados).

- Como orientación general solamente se indicará un tratamiento con ortesis craneal en los casos de grado grave en los que la plagiocefalia se acompañe de clara deformación craneofacial, habiéndose ya agotado la posibilidad de tratamiento postural.

\section{Discusión}

Este protocolo ha sido diseñado a petición de la Administración Sanitaria de la Comunidad de Madrid.

Por ello el objetivo principal de este trabajo es clarificar lo más posible la confusión existente en esta patología, publicando además un protocolo de asistencia que puede ser aplicado no solamente en la Comunidad de Madrid, sino en cualquier tipo de red sanitaria pública. Es en nuestra opinión, el primer protocolo de este tipo que se publica en la literatura.

Creemos que define bien las funciones de los diferentes especialistas así como los tiempos del proceso según la edad del niño. La elección de la fecha límite de 5 meses de edad está fundamentada en dos razones: la ortesis craneal comienza en general a aplicarse a los 4-5 meses de edad. Por otra parte, en el caso de las craneosinostosis occipitales, la edad en la que comienza el tiempo adecuado para la cirugía es también la misma, por lo que ambas edades son las más adecuadas en los casos de que el pediatra tenga que enviar el niño al neurocirujano.

Sin duda, la fisiopatología de estas deformaciones no está todavía bien explicada, especialmente en relación con las diferencias existentes entre las craneosinostosis y las plagiocefalias no craneosinostóticas. Esta es, seguramente, la razón principal de la confusión que existe en la literatura.

Incluso algunos autores han propuesto un origen fisiopatológico común para ambas entidades: según Dias et $\mathrm{al}^{9,10}$, las fuerzas mecánicas que provocan la deformación primitiva de la región occipital pueden originar, si su actuación es muy persistente, cambios patológicos en las suturas lambdoideas y la base craneal al final del proceso, transformándose en auténticas craneosinostosis.

Hay que tener en cuenta que factores que impliquen inmovilización y compresión de suturas similares han sido también implicados para explicar algunas formas de craneosinostosis, como las sagitales y metópicas ${ }^{11}$ (Fig. 11).

Por todo lo anterior, las suturas pueden aparecer patológicas en ambos procesos ${ }^{16,27}$ y entonces el diagnóstico diferencial puede ser difícil. Esta es la razón por la que
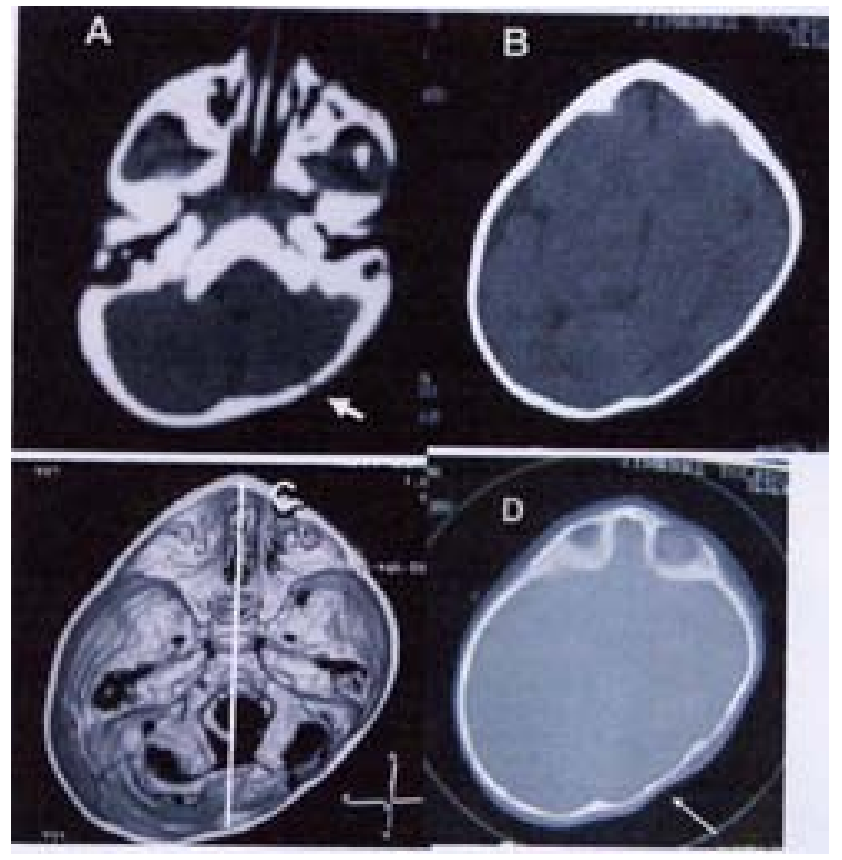

Fig.11.- A y B: Tac intracraneal. C: Reconstrucción tridimensional.

Niño con trigonocefalia y plagiocefalia posicional derecha. A: Sutura lambdoidea derecha permeable (flecha). B: Aplanamiento occipital derecho. Cresta metódica evidente y región frontal de aspecto triangular (típicos de trigonocefalia). C: Eje antero-posterior basal sin desviaciones. D: Irregularidades en la tabla interna de la región.

en nuestro protocolo no hemos indicado ningún estudio radiológico en la fase pediátrica de tratamiento, puesto que para realizarlo es necesario una TAC craneal y la interpretación de un experto en deformaciones craneales.

La plagiocefalia posicional puede acompañarse también de muchas otras lesiones. Sin duda, el tortícolis es la más frecuente. Esta contractura muscular de algunos músculos del cuello puede ser provocada por fibrosis de los esternocleidomastoideos producida como resultado de traumatismos en el parto, también por tumores de los propios músculos, pero actualmente se ha demostrado que la asimetría de los cóndilos occipitales es muy frecuente en las plagiocefalias, siendo, probablemente, ésta la razón más importante y frecuente para el desarrollo de tortícolis ${ }^{22}$, así como de algunas alteraciones de los músculos oculares.

La plagiocefalia puede también acompañarse de muy variadas lesiones del sistema nervioso central tal como se ha comentado anteriormente (macrocefalia, acúmulos de LCR extraaxiales, hidrocefalia, hemorragia perinatal, infecciones, espina bífida, e incluso retrasos en el aprendizaje o psicomotores.

Recientemente algunos autores ${ }^{14}$ han obtenido según 
ellos buenos resultados como consecuencia del tratamiento con “cascos” en relación a estos retrasos psicomotores.

Los autores de este trabajo creen que no existe base científica para ello, estando más de acuerdo con otros autores como Barlett, quien explica claramente en su crítica ${ }^{4}$ que es lógico pensar que los daños neurológicos previos al desarrollo de una plagiocefalia son un factor predisponente de primer orden para que se produzca posteriormente una plagiocefalia posicional, entre otras razones por la menor movilidad que pueden tener estos niños.

Finalmente, pensamos, como muchos otros autores, que el tratamiento de la plagiocefalia posicional debe realizarse de manera razonablemente escalonada ${ }^{2021,24,25}$, tal como hemos desarrollado en el protocolo presentado en este trabajo. Esto, y lograr además una coordinación lógica entre pediatras, rehabilitadores y neurocirujanos, constituye la base de nuestro protocolo.

Esto es todavía más necesario si pensamos que el tratamiento con ortesis craneal está siendo aplicado en nuestra opinión de una forma excesiva, sin tener en cuenta las grandes posibilidades que tiene el tratamiento postural y rehabilitador.

Independientemente de si existe o no financiación pública para la ortesis craneal, la elaboración y utilización de un protocolo coordinado es cada vez más necesario.

\section{Bibliografía}

1. American Academy of Pediatrics, Task force on Positioning and Sudden Infant Death Syndrome. Positionings and SIDS. Pediatrics. 1992; 89: 1120-1126.

2. American Academy of Pediatrics, Task Force on Infant Sleep Positional Sudden Infant Death Syndrome. Changing concepts of sudden infant death syndrome: implications for infant sleeping environment and sleep position. Pediatrics 2000; 105: 650-656.

3. Argenta, L.C., David, L.R.,, Wilson, J.A.: An increase in infant cranial deformity with supine sleeping position. $\mathrm{J}$ Craniofac Surg 1996; 7: 5-11.

4. Barlett, S.P.: Neurodevelopment delays in children with deformational plagiocephaly. Discussion. Plastic and Reconstructive Surgery 2006; 117: 219-220.

5. Bialocerkowski, A.E., Viadusic, S.L., Howell, SM.: Conservative interventions for positional plagiocephaly: a systematic review. Dev Med Chil Neurol. 2005; 47: 563570 .

6. Capon-Degardin, N., Arnaud, E., Marchac, D., Reñiré, D.: La plagiocephalie posturale ou plagiocephalie d'origen positionnelle. Pédiatrique Pratique. 2004; nº 1155fébrier.

7. Chadduck, W.M., Kast, Jh., Donahue, D.J.: The enigma of lambdoid positional molding. Pediatr Neurosurg 1997; 26: 304-311.
8. Czorni, A., Yettou, H., Forlodou, P., Striker, M.: L'arrière-crâne. Classification des dismorphies. Therapeutic original: la transposition bilaterale retournèe. Neurochirurgie 1995; 41 (suppl4): 295-314.

9. Dias, M.S., Klein, D.M.: Occipital plagiocephaly: deformation or lambdoid synostosis? A unifying theory regarding pathogenesis. Pediatric Neurosurgery 1996; 24: 69-73.

10. Dias, M.S., Klein, D.M., Backstrom, J.W.: Occipital plagiocephaly: deformation or lambdoid synostosis? Morphometric analysis and results of unilateral lambdoid craniectomy. Pediatric Neurosurgery 1996; 24: 61-68.

11. Graham, J.M., de Saxe, M., Smirth, D.W.: Sagital craniostenosis: Fetal head constraint as one posible cause. J Pediatr 1979; 95: 747-750.

12. Huang, M.H., Gruss, J.S., Clarren, S.K.: The differential diagnosis of posterior plagiocephaly: True lamndoid synostosis versus positional molding. Plastic and Reconstructive Surg 1996, October 765-774.

13. Kane, A.A., Mitchell, L.E., Craven, K.P.: Observations on a recent increase of plagiocephaly whitout synostosis. Pediatrics 1996, 97; 877-885.

14. Kordestani, R.K., Patel, S., Bard, E., Gurwich, R., Panchal, J.: Neurodevelopment delays in children with deformational plagiocephaly. Surgery 2006; 117: 207218.

15. Littlefield, T.R., Kelly, K.M., Pomatto., J.K.:Multiple-birth infants at higher risk for development of deformational plagiocephaly. Pediatrics 1999; 103: 565-560.

16. Losee, J.E., Feldman, B.S., Ketkar, M.: Nonsynostotic occipital plagiocephaly: radiographic diagnosis of the "sticky suture". Plastic and Reconstr Surg 2005; 116: 1860-1869.

17. Martínez-Lage, J.F., Ruiz-Espejo, A.M., Gulabert, A., Perez-Espejo, M.A., Guillén-Navarro, E.: Positional skull deformities in children: skull deformation without synostosis. Child’s Nerv Syst. 2006; 22: 368-374.

18. Mernard, R.M., David, D.J.: Unilateral lambdoidsynostosis:morphological characteristics. J Craniofac Surg 1998; 9: 240-246.

19. Miller, R.I., Clarren, S.K.: Long-term development outcomes in patients with deformational plagiocephaly. Pediatrics. 2000; 105: 1-5.

20. Morrison, C.S., Chariker, M.: Positional plagiocephaly: pathogenesis, diagnosis and management. J Ky Med Assoc. 2006; 104: 136-140.

21. Moss, S.D.: Non surgical, non orthotic treatment of occipital plagiocefhaly. What is the natural history of the misshapen neonatal head? J Neurosurg 1997; 87: 667-670.

22. Mottolese, C., Szathmari, A., Ricci, A.C.: Plagiocephalies positionnelles: place de l'orthese crânienne. Neurochirurgie 2006; 52: 184-194.

23. Muñoz, Ma.J., Esparza, J., Hinojosa, J., Romance, 
A.: Plagiocefalia posterior postural. Tratamiento remodelador externo. Neurocirugía 2000; 11: 364-372

24. Persing, J., James, H., Swanson., et al.: Prevention and management of positional skull deformities in infants. Pediatrics 2003; 112: 199-202.

25. Pollack, I.F., Losken, W.H., Fasik, P.: Diagnosis and management of posterior plagiocepgaly. Pediatrics 1997; 99: 180-185.

26. Rekate, H.L.: Occipital plagiocephaly: a critical review of the literature. J Neurosurg 1998; 89: 24-30.

27. Sze, R.W., Hopper, R.A., Ghioni, V.: MDCT Diagnosis of the Child with posterior plagiocephaly. AJR 2005; 185: 1342-1346.
28. Turk, A.E, McCarthy, J.G., Thorne, C.H.: The back to sleep campaign and deformational plagiocephaly is the cause for concern? J Craneofac Surg 1996; 7: 1218.

Esparza, J.; Hinojosa, J.; Muñoz, Ma.J.; Romance, A.; García-Recuero, I.; Muñoz, A.: Diagnóstico y tratamiento de la plagiocefalia posicional. Protocolo para un Sistema Público de Salud. Neurocirugía 2007; 18: 457-467.

Correspondencia postal: Dr. Javier Esparza. Servicio de Neurocirugía Pediátrica. Hospital Infantil 12 de Octubre. Avda. de Córdoba s/n. 28041 Madrid. 